\title{
EFECTOS ASOCIADOS AL CAMBIO DEL CAUCE DEL RÍO SAN LORENZO, EL CARMEN DE VIBORAL-ANTIOQUIA
}

\author{
Jorge Wilson Naranjo Cardona ${ }^{1}$, Yimmy Montoya-Moreno ${ }^{2}$
}

${ }^{1}$ Magister en Ciencias Naturales y Matemática Universidad Pontificia Bolivariana, Medellín, Colombia, email:wjnaranjo@gmail.com.

2Doctor en Ingeniería, Investigador Grupo Geolimna, Universidad de Antioquia, docente Universidad Pontificia Bolivariana e-mail: yimmymontoya3@hotmail.com.

\section{RESUMEN}

El objeto de investigación en este proyecto giró en torno a los cambios que sufre el cauce de un río al ser modificado su curso. Para ello se llevó a cabo un trabajo de muestreo en la cuenca hidrográfica del río San Lorenzo, ubicado en el Municipio de El Carmen de Viboral, Oriente Antioqueño, teniendo en cuenta variables fisicoquímicas y bioindicadores como los macroinvertebrados bentónicos. Se corroboró que la desviación y/o cambio del cauce del río San Lorenzo, ha provocado modificaciones en sus características fisicoquímicas y biológicas, evidenciadas en la disminución de la concentración y saturación del oxígeno disuelto, aumento del pH (debido al incremento en la cobertura del perifiton y la fotosíntesis que este genera) y la temperatura del agua (el agua casi no presenta flujo, por lo que se incrementa su energía cinética). Respecto a los macroinvertebrados acuáticos se registró una reducción fuerte de la riqueza y diversidad respecto al cauce normal del río. También se encontró un cambio en la estructura de la comunidad de los macroinvertebrados menos resistentes a la contaminación por otros más tolerantes, pérdida de biodiversidad acuática y un incremento en la concentración de fósforo en el agua derivado de los desechos de una truchera.

Palabras clave: Desvío de cauce, disturbio, macroinvertebrados acuáticos, enriquecimiento de fósforo.

Recibido: 31 de Marzo de 2020. Aceptado: 16 de Diciembre de 2020

Received: March 31, 2020. Accepted: December 16, 2020

\section{EFFECTS ASSOCIATED BY THE CHANGE OF CAUCE RÍO SAN LORENZO, CARMEN DE VIBORAL ANTIOQUIA}

\begin{abstract}
Abstract The object of investigation in this project revolves around the changes that the riverbed undergoes when its course is modified. For this, a sampling work will be carried out in the San Lorenzo river basin, located in the Municipality of El Carmen de Viboral, Oriente Antioqueño, taking into account physicochemical variables and bioindicators such as benthic macroinvertebrates. It was confirmed that the deviation and / or change of the San Lorenzo river channel has caused changes in its physicochemical and biological characteristics, evidenced in the decrease in the concentration and saturation of dissolved oxygen, increase in $\mathrm{pH}$ (due to the increase in the coverage of the periphery and the photosynthesis that this generates) and the temperature of the water (the water almost does not present flow, reason why its kinetic energy is increased). Regarding aquatic macroinvertebrates, there was a strong reduction in wealth and diversity compared to the normal riverbed. A change was also found in the community structure of macroinvertebrates less resistant to contamination by more tolerant ones, loss of aquatic biodiversity and an increase in the concentration of phosphorous in the water derived from the waste of a trout.
\end{abstract}

Keywords: Channel diversion, disturbance, aquatic macroinvertebrates. phosphorus enrichment.

Cómo citar este artículo: J. Naranjo, Y. Montoya. "Efectos asociados al cambio del cauce del río San Lorenzo, el Carmen de Viboral-Antioquia”, Revista Politécnica, vol.16, no.32 pp.112-128, 2020. DOI:10.33571/rpolitec.v16n32a11 


\section{INTRODUCCIÓN}

El agua es esencial para el mantenimiento de todos los procesos biológicos, la calidad de vida de una sociedad y el sostenimiento de las actividades económicas. La calidad del agua está definida por su composición química y por sus características físicas, adquiridas a través de procesos naturales y antropogénicos que limitan o perjudican su uso. Este es evaluado al comparar los valores que asumen los parámetros indicadores con estándares y criterios establecidos. La variación espacio temporal de la calidad se modifica por el influjo de múltiples actividades socioeconómicas y naturales, y la intensidad de esta variación es determinada por las características propias de estas dinámicas [1].

Es claro que durante el último siglo se han llevado a cabo numerosas actuaciones con fines de regulación hidrológica (embalses), de restauración hidrológico-forestal (repoblaciones, diques de retención), de protección contra inundaciones (motas, malecones, encauzamientos), de explotación de recursos (canteras), etcétera, que han alterado profundamente aguas abajo el balance de agua y sedimento y, con ello, la morfología de los cauces [2]. En definitiva, toda una serie de perturbaciones introducidas por el hombre en un sistema ambiental de frágil equilibrio como es el fluvial.[3] refiere que la construcción de represas, bien sea para proyectos hidroeléctricos, suministro de agua o control de inundaciones, ha causado impactos en el campo de la biología y ecología de los mismos ríos (pérdida de hábitat, nichos, freno a la migración de peces y otros).

Las alteraciones morfológicas son alteraciones de la forma del río. Suponen la modificación total o parcial de los sistemas fluviales. Entrarían aquí actuaciones como defensas, dragados, extracciones de áridos, rectificaciones de cauce, canalizaciones, urbanización y construcción de estructuras en las márgenes [4]. Dichas modificaciones y actividades, "generan efectos graves en la disponibilidad espacio - temporal de la oferta hídrica, lo que deteriora las condiciones fisicoquímicas y biológicas del agua” [5].

Estudios realizados en diferentes ríos, evidencian impactos a partir de la modificación en la morfología del cauce por causas de tipo antrópico [1], [2], [5]. Dichos impactos se ven reflejados en los componentes fisicoquímicos y biológicos del agua que son a su vez un indicador importante. [2] infieren que los cambios originados aguas arriba influyen aguas abajo con un retardo temporal causa efecto, especialmente en los tramos medio e inferior de los cursos fluviales.

En la actualidad, comunidades biológicas como las de macroinvertebrados bentónicos, han sido incluidas como indicadoras de las condiciones ambientales de ecosistemas acuáticos, ya que su prevalencia en el ambiente en el que viven, refleja niveles de presión antrópica sobre los mismos. [3], [6], [7]. Para el ecólogo, un ecosistema acuático es un sistema funcional en el cual hay un intercambio cíclico de materia y energía entre los organismos vivos y el ambiente abiótico. Por lo tanto, la biología y la química, están estrechamente relacionadas; en la evaluación de las aguas naturales y contaminadas juegan papeles complementarios. [8].

Al tener en cuenta el papel fundamental que desempeñan los macroinvertebrados en los sistemas fluviales, se tiene como objetivo evaluar la presencia de dichos organismos a partir de una comparación espacial y temporal de las comunidades biológicas del río San Lorenzo, a partir de la desviación del cauce para la implementación de estanques con fines productivos.

\section{MATERIALES Y METODOS}

La cuenca hidrográfica del Río San Lorenzo está ubicada en jurisdicción del municipio de El Carmen de Viboral, el cual está situado al suroriente del Departamento de Antioquia, en la subregión Valles de San 
Nicolás, con coordenadas geográficas de 6은 $09^{\prime \prime}$ de latitud norte y 7520'19" longitud Oeste, a una altura sobre el nivel del mar de $2150 \mathrm{~m}$, y con una temperatura promedio de $17^{\circ} \mathrm{C}$ [10].

En el río San Lorenzo se ubicaron seis (6) estaciones de muestreo en puntos estratégicos observados directamente en campo; Estación 1 (E1) antes de la desviación del cauce para la implementación de los estanques piscícolas (condiciones naturales y línea base de comparación con el resto). Estación 2 (E2) remanente de agua del canal pluvial por donde pasaba el cauce natural antes de la intervención (corredor hiporréico). Estación 3 (E3) después del retorno del agua que sale de los estanques al cauce natural del río (salida de las trucheras). Estación 4 (E4) aguas abajo al lado de la escuela de la vereda San Lorenzo. Estación 5 (E5) en donde recibe un tributario de bajo orden (turbio). Estación 6 (E6) punto sobre la zona del puente que comunica hacia la escuela de San Lorenzo y sitio posible para una futura bocatoma del acueducto municipal de El Carmen de Viboral (Figura 1 - 2).

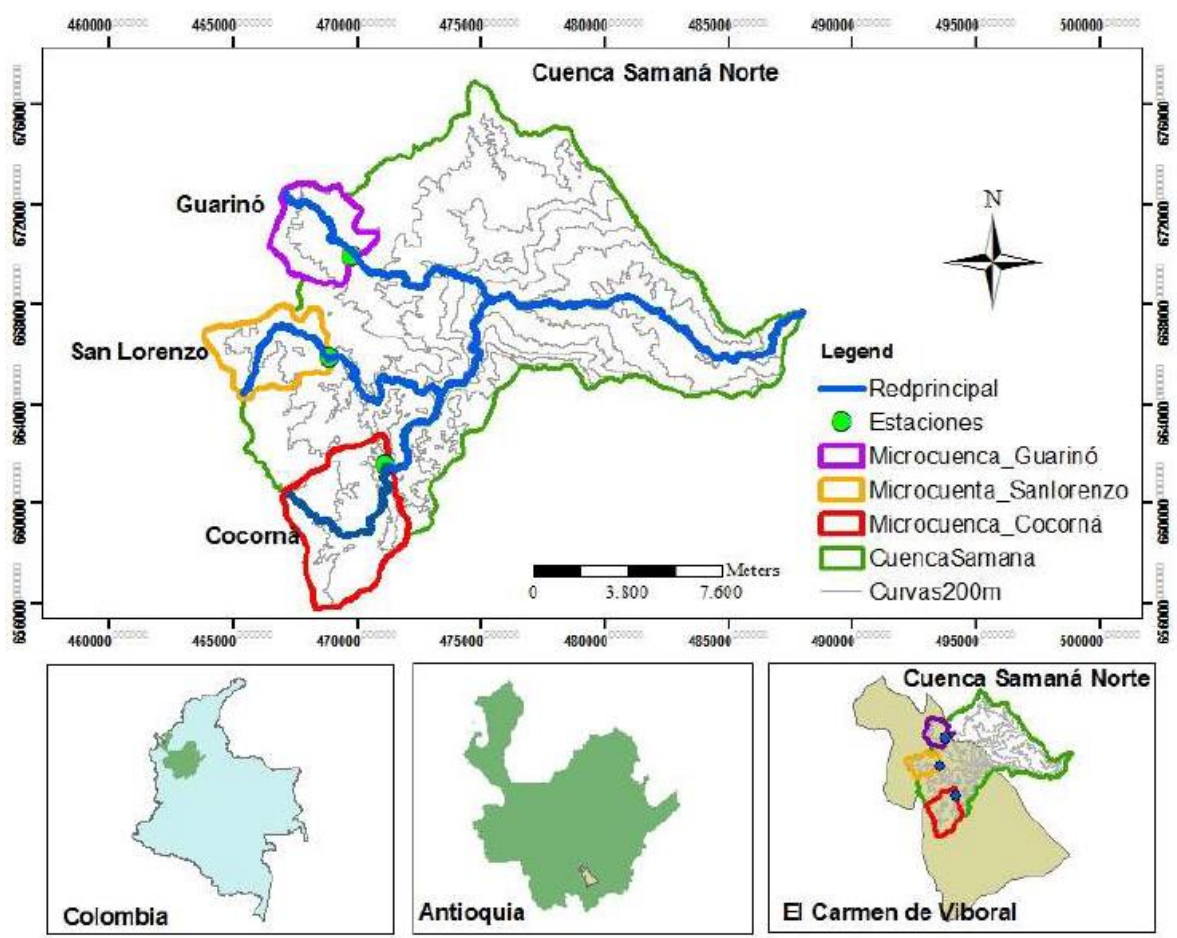

Figura 1. Ubicación espacial del Río San Lorenzo (Tomado de Botero-Álvarez, 2019) 


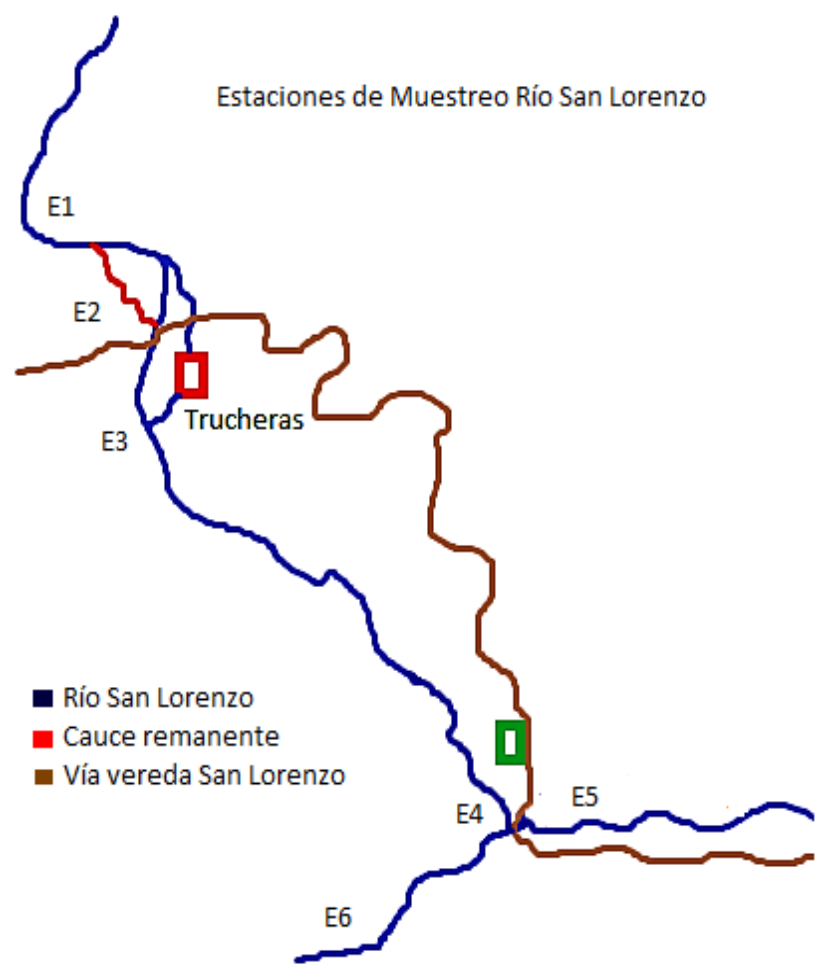

Figura 2. Ubicación espacial de las estaciones.

\section{Muestreo}

Para la medición de las variables fisicoquímicas del agua y la recolección de las muestras biológicas, cada estación fue visitada en cuatro ocasiones durante los meses de noviembre de 2018, febrero, marzo y mayo de 2019 en diferentes momentos pluviométricos.

\section{Variables fisicoquímicas}

Para determinar la calidad fisicoquímica del agua, estos sitios de muestreo fueron visitados durante periodos hidrológicos diferentes en una ventana temporal de siete (7) meses con una periodicidad bimensual y se registraron in situ parámetros como el pH, conductividad eléctrica, oxígeno disuelto, porcentaje de saturación de oxígeno, temperatura del agua, sólidos totales disueltos evaluados mediante un equipo multiparámetro WTW.

Se tomaron muestras de agua en cada estación para el análisis en el laboratorio de agua de la facultad de Ingeniería de la Universidad de Antioquia, en el que se evaluaron los siguientes parámetros: nitratos, nitritos, fósforo soluble reactivo y hierro por medio de métodos colorimétricos y espectrofotométricos.

\section{Macroinvertebrados bentónicos}

Los macroinvertebrados fueron colectados a contracorriente por medio de una red, con réplica en cada lugar de muestreo y se complementó con recolecciones manuales de organismos adheridos a las piedras mediante pinzas. Los especímenes colectados fueron depositados en una solución de alcohol al $90 \%$ en recipientes de plásticos debidamente rotulados con fecha y lugar de recolección, para su posterior identificación a nivel de familia. Esta colecta, permitió realizar un análisis cuantitativo de las morfoespecies de macroinvertebrados con respecto a su riqueza de taxa y abundancia en el sitio de colección a partir de claves taxonómicas especializadas [11], [3], [12], [13], [14]. Con el listado de familias reportadas se calculó el índice BMWP/Col. [6] 


\section{Análisis de datos}

Los datos obtenidos en los 4 muestreos fueron sometidos a un análisis exploratorio mediante el uso de estadísticos de tendencia central (media aritmética) y de dispersión (coeficiente de variación relativa de Pearson, C.V). Se realizó un análisis de correspondencia canónica rectificado (DCCA) para identificar la longitud del gradiente y luego se hizo un análisis de redundancia canónica (RDA) en donde se integraron los géneros más importantes, las variables evaluadas y los sitios de colecta, para identificar los patrones de distribución de los macroinvertebrados acuáticos. Los análisis estadísticos mencionados se efectuaron con el paquete estadístico Statgraphics Centurion XV y Canoco 5.0. Los índices comunitarios se calcularon mediante el paquete PAST.

\section{Índices}

En los análisis de las comunidades de macroinvertebrados bentónicos, se aplicaron índices ecológicos, que involucraron cuatro elementos sobre las estructuras de las comunidades bióticas: riqueza, equidad, dominancia y abundancia. Adicional a estos índices se calculó el índice BMWP/Col. [6] para evaluar la calidad biológica del agua en las estaciones de muestreo.

\section{RESULTADOS}

\section{Características Climáticas y Fisicoquímicas:}

Se realizaron cuatro muestreos durante el ciclo hidrológico que comprende un período húmedo durante el mes de noviembre de 2018, seguidamente, un periodo seco entre los meses de febrero y marzo, y finalmente, un regreso de la temporada húmeda durante el mes de mayo de 2019 en donde se obtienen las últimas muestras.

Las variables fisicoquímicas registradas en el Río San Lorenzo durante los muestreos realizados en las diferentes estaciones presentaron las siguientes variaciones: la temperatura del agua $\left({ }^{\circ} \mathrm{C}\right)$ presentó una temperatura inferior $\left(15^{\circ} \mathrm{C}\right)$ en la estación E3 (salida de truchera) en el mes de marzo y una temperatura mayor $\left(20,5^{\circ} \mathrm{C}\right)$ en la misma estación en el mes de febrero. El pH mostró un valor mayor de 7,99 en la estación E5 (afluente turbio) en el mes de febrero y un valor inferior de 6,2 en la estación E2 (corredor hiporréico) en los meses de febrero y mayo. El oxígeno disuelto (ppm) presentó una concentración alta de 7,83 en la estación E3 en el mes de noviembre de 2018 y una concentración baja de 3,92 en la estación E2 en el mes de mayo. La Saturación de oxígeno (\%) fue sobresaturada con un nivel alto de $105,5 \%$ en la estación E1 (antes de derivación) en los meses de noviembre y marzo y un valor subsaturado de $51,7 \%$ en la estación E2 en el mes de marzo. La Conductividad eléctrica $(\mu \mathrm{S} / \mathrm{cm})$ registró un valor alto de 31 en la estación E2 en el mes de febrero y un mínimo valor mínimo de 15 en la estación E4 (claro) en el mes de mayo. Los Sólidos Totales Disueltos (STD) (mg/l) presentaron un valor alto de 35 en la estación E2 en el mes de febrero y un valor mínimo de 16 en las estaciones E5 en el mes de noviembre y E1 y E4 en el mes de mayo. La concentración de $\mathrm{NO}_{3}(\mathrm{mg} / \mathrm{l})$ presentó un valor mayor de 2,8 en la estación E6 (unión) en los meses de febrero y mayo y un valor inferior de 0,01 en la estación E4 en los meses de noviembre y marzo. La concentración de $\mathrm{NO}_{2}$ (mg/l) fue mayor en la estación E2 con un valor de 0,009 en los meses de noviembre y marzo y menor en la estación E5 con un valor de 0,001 en los mismos meses. La concentración de $\mathrm{PO}_{4}$ (mg/l) presentó valores altos de 6,72 en la estación E5 en los meses de noviembre y marzo y valores bajos de 0,06 en la estación E6 en los mismos meses. Finalmente, la concentración de Fe (mg/l) presentó valores elevados en la estación E2 con valores de 2,9 en los meses de noviembre y marzo y valores mínimos en la estación E4 con valores de 0,11 en los meses de febrero y mayo (Tabla 1). 
Tabla 1. Variables fisicoquímicas registradas en seis estaciones en cuatro periodos hidrológicos del año

\begin{tabular}{|c|c|c|c|c|c|c|c|c|c|c|c|}
\hline Muestreo & Estación & $\begin{array}{l}\mathrm{pH} \text { (uni- } \\
\text { dades de } \\
\mathrm{pH})\end{array}$ & $\begin{array}{l}\text { Oxígeno } \\
\text { disuelto } \\
\text { (ppm) }\end{array}$ & $\begin{array}{l}\text { Saturación de } \\
\text { oxígeno (\%) }\end{array}$ & $\begin{array}{c}\text { Temperatura } \\
\text { del agua } \\
\left({ }^{\circ} \mathrm{C}\right)\end{array}$ & $\begin{array}{c}\text { Conductividad } \\
\text { eléctrica } \\
(\mu \mathrm{S} / \mathrm{cm})\end{array}$ & $\begin{array}{c}\text { Sólidos Tota- } \\
\text { les Disueltos } \\
\text { (mg/l) }\end{array}$ & $\begin{array}{l}\mathrm{NO} 3 \\
(\mathrm{mg} / \mathrm{l})\end{array}$ & $\begin{array}{l}\mathrm{NO} 2 \\
(\mathrm{mg} / \mathrm{l})\end{array}$ & $\begin{array}{c}\mathrm{PO} 4 \\
(\mathrm{mg} / \mathrm{l})\end{array}$ & $\begin{array}{c}\mathrm{Fe} \\
(\mathrm{mg} / \mathrm{l})\end{array}$ \\
\hline 1 & 1 & 7,678 & 7,82 & 105,5 & 16,3 & 21,5 & 21 & 0,04 & 0,002 & 0,87 & 0,31 \\
\hline 1 & 2 & 6,4 & 4,86 & 64,2 & 15,8 & 29,9 & 32 & 0,12 & 0,009 & 1,2 & 2,9 \\
\hline 1 & 3 & 7,01 & 7,83 & 105,2 & 16,2 & 21,5 & 22 & 0,03 & 0,005 & 0,08 & 1,3 \\
\hline 1 & 4 & 6,39 & 6,96 & 94,8 & 16,2 & 25,4 & 26 & 0,01 & 0,002 & 3,4 & 0,3 \\
\hline 1 & 5 & 7,35 & 7,66 & 103,2 & 16,2 & 16,3 & 16 & 0,02 & 0,001 & 6,72 & 0,23 \\
\hline 1 & 6 & 6,7 & 7,67 & 103,5 & 16,1 & 20 & 20 & 0,04 & 0,004 & 0,05 & 0,32 \\
\hline 2 & 1 & 7,23 & 7,62 & 104,2 & 16,7 & 19,2 & 19 & 2,6 & 0,006 & 1,86 & 0,24 \\
\hline 2 & 2 & 6,2 & 4 & 56 & 18,7 & 31 & 35 & 0,4 & 0,006 & 0,92 & 0,15 \\
\hline 2 & 3 & 6,91 & 6,93 & 103 & 20,5 & 23,8 & 24 & 1,1 & 0,008 & 0,24 & 0,2 \\
\hline 2 & 4 & 7,05 & 7,54 & 103,6 & 16,5 & 27,8 & 28 & 1,2 & 0,007 & 0,45 & 0,11 \\
\hline 2 & 5 & 7,99 & 7,53 & 102,5 & 16,6 & 17,4 & 18 & 2,4 & 0,006 & 0,09 & 0,3 \\
\hline 2 & 6 & 6,79 & 7,58 & 102,7 & 16,5 & 20,8 & 21 & 2,8 & 0,002 & 0,09 & 0,14 \\
\hline 3 & 1 & 7,678 & 7,82 & 105,5 & 16,3 & 21,5 & 21 & 0,04 & 0,002 & 0,87 & 0,31 \\
\hline 3 & 2 & 6,25 & 3,93 & 51,7 & 15,4 & 24,1 & 24 & 0,12 & 0,009 & 1,2 & 2,9 \\
\hline 3 & 3 & 7,01 & 6,66 & 103,1 & 15 & 22,8 & 23 & 0,03 & 0,005 & 0,08 & 1,3 \\
\hline 3 & 4 & 6,96 & 7,51 & 101,1 & 16 & 25 & 26 & 0,01 & 0,002 & 3,4 & 0,3 \\
\hline 3 & 5 & 7,31 & 7,53 & 101,2 & 15,9 & 18 & 18 & 0,02 & 0,001 & 6,72 & 0,23 \\
\hline 3 & 6 & 6,93 & 7,53 & 101,2 & 15,9 & 21 & 21 & 0,04 & 0,004 & 0,05 & 0,32 \\
\hline 4 & 1 & 7,2 & 7,64 & 101,6 & 15,8 & 16,5 & 16 & 2,6 & 0,006 & 1,86 & 0,24 \\
\hline 4 & 2 & 6,2 & 3,92 & 51,8 & 15,5 & 22,9 & 23 & 0,4 & 0,006 & 0,92 & 0,15 \\
\hline 4 & 3 & 6,8 & 7,12 & 94,9 & 16,1 & 17,5 & 18 & 1,1 & 0,008 & 0,24 & 0,2 \\
\hline 4 & 4 & 7,17 & 7,58 & 101,1 & 15,7 & 15 & 16 & 1,2 & 0,007 & 0,45 & 0,11 \\
\hline 4 & 5 & 6,97 & 7,68 & 102,5 & 15,7 & 22,9 & 23 & 2,4 & 0,006 & 0,09 & 0,3 \\
\hline 4 & 6 & 6,81 & 7,63 & 101,7 & 15,7 & 19,8 & 20 & 2,8 & 0,002 & 0,09 & 0,14 \\
\hline
\end{tabular}


Según los datos registrados, se encontraron niveles bajos en la estación E2 (corredor hiporréico) con relación al pH $(6,2)$ en los meses de febrero y mayo; el oxígeno disuelto (ppm) presentó concentración baja $(3,92)$ en el mes de mayo; la saturación de oxígeno (\%) mostró un porcentaje bajo $(51,7)$ en el mes de marzo; la Conductividad eléctrica $(\mu \mathrm{S} / \mathrm{cm})$ registró un valor alto (31) en el mes de febrero; los Sólidos Totales Disueltos (STD) (mg/l) registraron un valor alto (35) en el mes de febrero; la concentración de $\mathrm{NO}_{2}(\mathrm{mg} / \mathrm{l})$ fue mayor $(0,009)$ en los meses de noviembre y marzo; el último registro de las variables que se tomaron, fue la concentración de Fe (mg/l) que presentó un valor alto $(2,9)$ en los meses de noviembre y marzo. Todos estos rangos de valores muestran el grado de afectación de la estación corredor hiporréico en comparación con las demás estaciones de muestreo.

Con respecto al coeficiente de variación de las variables fisicoquímicas, las podemos agrupar en los siguientes grupos: las poco variables ( $\mathrm{CV}<20 \%)$ tales como el $\mathrm{pH}$, el oxígeno disuelto, el \% de saturación, la temperatura del agua y la conductividad eléctrica; las de variación intermedia $(20 \%>C V<50 \%)$ como los sólidos totales disueltos; y las variables de variación alta $(\mathrm{CV}>50 \%)$, tales como los nitratos, los nitritos, fosfatos y el hierro (Tabla 2).

Las condiciones fisicoquímicas del agua indican que las aguas del Río San Lorenzo son frías, con pH circum-neutral, oxigenadas con saturación alta, aguas poco mineralizadas (conductividad eléctrica y sólidos totales bajos), con bajas concentraciones de nitratos, nitritos e intermedia para el fósforo soluble reactivo.

Tabla 2. Medidas de tendencia central y de dispersión de las variables físicas.

\begin{tabular}{|l|l|l|l|l|}
\hline Variables & Media & Máximo & Mínimo & $\begin{array}{l}\text { C.V } \\
(\%)\end{array}$ \\
\hline $\begin{array}{l}\text { Temperatura } \\
\text { del agua }\left({ }^{\circ} \mathrm{C}\right)\end{array}$ & 16,30 & 20,50 & 15,00 & 6,88 \\
\hline $\begin{array}{l}\mathrm{pH}(\mathrm{unidades} \\
\text { de pH) }\end{array}$ & 6,96 & 7,99 & 6,20 & 6,70 \\
\hline $\begin{array}{l}\text { Oxígeno di- } \\
\text { suelto (ppm) }\end{array}$ & 6,94 & 7,83 & 3,92 & 18,81 \\
\hline $\begin{array}{l}\text { Saturación de } \\
\text { oxígeno (\%) }\end{array}$ & 94,41 & 105,50 & 51,70 & 18,96 \\
\hline $\begin{array}{l}\text { Conductividad } \\
\text { eléctrica } \\
(\mu \mathrm{c} / \mathrm{cm})\end{array}$ & 21,73 & 31,00 & 15,00 & 19,06 \\
\hline $\mathrm{STD}(\mathrm{mg} / \mathrm{l})$ & 22,13 & 35,00 & 16,00 & 21,59 \\
\hline $\mathrm{NO}_{3}(\mathrm{mg} / \mathrm{l})$ & 0,90 & 2,80 & 0,01 & 121,02 \\
\hline $\mathrm{NO}_{2}(\mathrm{mg} / \mathrm{l})$ & 0,00 & 0,01 & 0,001 & 53,071 \\
\hline $\mathrm{PO}_{4}(\mathrm{mg} / \mathrm{l})$ & 1,33 & 6,72 & 0,05 & 144,03 \\
\hline $\mathrm{Fe}(\mathrm{mg} / \mathrm{l})$ & 0,54 & 2,90 & 0,11 & 145,70 \\
\hline
\end{tabular}

\section{Variables Biológicas:}

En la tabla 3 se presentan los macroinvertebrados recolectados mediante el método cuantitativo durante las campañas de toma de muestras realizadas en los meses de noviembre de 2018, febrero, marzo y mayo de 2019. En el Río San Lorenzo se recolectaron un total de 35 géneros pertenecientes a 27 familias y 1044 ejemplares de macroinvertebrados pertenecientes a los phylum platyhelminthes, annelidae, mollusca y arthropoda.

Del total de las taxas colectadas, la mayor riqueza corresponde a los tricópteros con seis (6) géneros, seguida por los dípteros y efemerópteros con cinco (5) géneros respectivamente y los haplotáxidos y basommato- 
foros con tres (3) géneros cada uno. Las familias que presentaron mayor abundancia fueron Haplotaxidae, Chironomidae, Simuliidae, Glossossomatidae y Naididae. En éstas se observó que, en la mayoría de las estaciones de muestreo durante el período hidrológico, la diferencia de esta distribución se presentó en la estación San Lorenzo corredor hiporréico (E2) en donde hubo una menor presencia de taxas.

En la estación E1 (antes de derivación) predominan los tricópteros Mortoniella sp (Glossossomatidae) seguidos por los dípteros Chironomidae (Chironomidae) y Simulium sp (Simuliidae). En la estación E2 corredor hiporréico la ausencia de taxas e individuos es un indicador del impacto generado por el cambio del cauce y la reducción del flujo.

En la estación E3 salida truchera predominan los tricópteros Mortoniella sp (Glossossomatidae) seguido por los dípteros Chironomidae (Chironomidae), Basommatophoros Physa sp (Physidae), Haplotaxida Naididae (Haplotaxidae) respectivamente. En la estación E4 claro no se encuentra un número representativo de individuos por familia. En la estación E5 afluente turbio predominan los dípteros Simulium sp (Simuliidae) seguido por los tricópteros Mortoniella sp (Glossossomatidae). En la estación E6 hubo una presencia importante de los tricópteros Mortoniella sp (Glossossomatidae) seguidos por los Dípteros Simulium sp (Simuliidae).

El mes de mayo de 2019 fue el mes que registró el mayor número de macroinvertebrados bentónicos colectados durante los muestreos hechos en las seis estaciones del Río San Lorenzo, seguido por el mes de marzo del mismo año. Es de resaltar la estación E1 antes de desviación en donde el número de individuos supera las otras cinco estaciones, lo cual se entiende a razón de que es la zona menos intervenida del sistema.

Frente a la distribución espacial y temporal las taxas Naididae, Chironomidae, Tanytarsini, Simulium y Mortoniella estuvieron presentes en la mayoría de las estaciones de muestreo durante la corriente del río San Lorenzo, a diferencia de la estación E2 corredor hiporreico en donde hubo una disminución del total de individuos colectados (sólo diecisiete 17 ejemplares) y de varias familias de macroinvertebrados acuáticos Chironomidae, Simulium sp, Gyraulus sp, Ferrisia sp, Microvelia sp y Mortoniella durante todo el tiempo de muestreos.

Algunas taxas como Collembola solo se encontraron en la estación San Lorenzo claro (E4), Naididae, Tabanidae, Helobdella y Staphylinidae en la estación San Lorenzo salida truchera (E3), Anchytarsus en la estación San Lorenzo Unión (E6), Baetidae y Acari sp2 en la estación San Lorenzo antes derivación (E1) y Moribaetis, Chimarra y Thraulodes en la estación San Lorenzo afluente turbio (E5).

En la Tabla 4 se pueden evidenciar variaciones en los valores de los índices comunitarios, la cantidad de Taxas e individuos y los valores del índice BMWP/Col en las diferentes estaciones de muestreo, en donde la estación con mayores valores de diversidad de macroinvertebrados, es la estación E1 con un valor mayor en el mes de mayo; mientras que la estación E2 corredor hiporréico presento valores inferiores durante todas las muestras, siendo el mes de febrero el más bajo.

En relación al índice de Dominancia de Simpson, los valores obtenidos en las diferentes campañas de muestreo presentaron variaciones en los valores obtenidos, los valores inferiores se encuentran en la estación E1 con un valor mínimo de 0.12 para el mes de noviembre de 2018 lo que se relaciona con mejor estabilidad ambiental de la zona y en la estación E4 se encontraron el valor máximo de 0.84 en el mes de febrero de 2019, lo que puede obedecer al impacto generado por la salida de las aguas de la explotación piscícola cargadas de nutrientes. 
Tabla 3. Macroinvertebrados acuáticos recolectados durante las campañas de toma de muestras.

\begin{tabular}{|c|c|c|c|c|c|c|c|c|c|c|c|c|c|c|c|c|c|c|c|c|c|c|c|c|c|c|}
\hline \multirow{2}{*}{ Orden } & \multirow{2}{*}{ Familia } & \multirow{2}{*}{ Género } & \multirow{2}{*}{\multicolumn{4}{|c|}{$\begin{array}{c}\text { San Lorenzo antes derivación } \\
\text { nov-18 feb-19 mar-19 mav-19 }\end{array}$}} & \multicolumn{4}{|c|}{ San Lorenzo corredor hiporreico } & \multicolumn{4}{|c|}{ San Lorenzo salida truchera } & \multicolumn{4}{|c|}{ San Lorenzo claro } & \multicolumn{3}{|c|}{ San Lorenzo Turbio } & \multicolumn{5}{|c|}{ San Lorenzo Unión } \\
\hline & & & & & & & nov-18 & feb-19 mal & & & nov-18 & feb-19 I & & may-19 1 & nov-18 & & mar-19 ma & $a y-19 \mathrm{nc}$ & $10 \mathrm{v}-18 \mathrm{fe}$ & eb-19 m & $\operatorname{mar}-19 \mathrm{ma}$ & & & & nar-19 m & ay-19 \\
\hline Arthopleona & Isotomidae & Collembola & & & & & & & & & & & & & & & & 2 & & & & & & & & \\
\hline \multirow{3}{*}{ Haplotaxida } & Tubificidae & Oligochaeta & & 1 & & 2 & & & & & 1 & & & 2 & & & 1 & 2 & & & 7 & 3 & & & & \\
\hline & Haplotaxidae & Naididae & 1 & 11 & 21 & 5 & & & & & 1 & 2 & 3 & 15 & & 1 & 2 & 1 & & & & 1 & 2 & 1 & & \\
\hline & Naididae & Naididae $s p$ & & & & & & & & & & & & 1 & & & & & & & & & & & & \\
\hline \multirow[t]{2}{*}{ Coleoptera } & Staphylinidae & Staphylinidae & & & & & & & & & & & 2 & & & & & & & & & & & & & \\
\hline & Ptilodactylidae & Anchytarsus sp & & & & & & & & & & & & & & & & & & & & & & & 1 & \\
\hline \multirow[t]{4}{*}{ Diptera } & Chironomidae & $\begin{array}{l}\text { Chironomidae } \\
\text { Chironomus }\end{array}$ & & 12 & 41 & 17 & 1 & 1 & & & 11 & $\begin{array}{l}3 \\
3\end{array}$ & $\begin{array}{r}22 \\
1\end{array}$ & 32 & & 1 & 1 & 11 & 1 & 10 & 4 & & & 4 & 1 & 2 \\
\hline & & Tanytarsini & & 1 & 2 & 2 & & & & & 1 & 2 & 1 & 5 & 5 & 5 & & & & & 1 & 2 & & 4 & 2 & 3 \\
\hline & Tabanidae & Tabanidae $s p$ & & & & & & & & & & & & 2 & & & & & & & & & & & & \\
\hline & Simuliidae & Simulium $s p$ & & 7 & 8 & 27 & 2 & & & 1 & 2 & 8 & & 4 & & 1 & 8 & 5 & 15 & 7 & & 1 & & 11 & 33 & 15 \\
\hline \multirow[t]{5}{*}{ Ephemeroptera } & Baetidae & Baetidae & & & & 1 & & & & & & & & & & & & & & & & & & & & \\
\hline & & Baetodes sp & & 2 & 4 & 8 & & & & & 1 & & & 5 & & & & & & & & & & & 1 & 1 \\
\hline & & Dactylobaetidae $s p$ & & & & & & & & & & 2 & & & & & & & & & & & & & & \\
\hline & & Moribaetis sp & & & & & & & & & & & & & & & & & 1 & & & & & & & \\
\hline & Leptophebiidae & Thraulodes $s p$ & & & & & & & & & & & & & & & & & 2 & & & & & & & \\
\hline Gastropoda & Planorbidae & Gyraulus sp & & & & & 2 & & & 1 & & & & & & & & & & & 1 & & & & & \\
\hline \multirow[t]{3}{*}{ Basommatophor: } & Ancylidae & Ferrisia $s p$ & & & & & 1 & 1 & & 1 & & & & & & & & & & & & & & & & \\
\hline & Physidae & Physa sp & & & & & & & & & 2 & 21 & 6 & & & & 9 & 5 & & 3 & 10 & & & 14 & 1 & \\
\hline & Lymnaeidae & Lymnaeidae $s p$ & & & & & & & & & & & 1 & & 1 & & & & & & & & & & & \\
\hline Hemiptera & Veliidae & Microvelia $s p$ & & & & & & & 5 & & & & & & & & & & 1 & & & & 5 & & & \\
\hline Lepidoptera & Pyralidae & sin identificar & & & 1 & & & & & & & & & & & & & & & & & & & & & \\
\hline Odonata & Calopterygidae & Hetaerina americana & & 3 & 1 & 1 & & & & & & & & & & & 2 & 1 & & & & 1 & & & & \\
\hline \multirow[t]{2}{*}{ Trombidiformes } & Hydrachnidiae & Acari spl & & 1 & & 1 & & & & & & & & 1 & & 1 & & & & & & & & & & \\
\hline & & Acari $s p 2$ & & & & 1 & & & & & & & & & & & & & & & & & & & & \\
\hline \multirow[t]{2}{*}{ Rhynchobdellida } & Glossiphoniidae & sin identificar & & & & & & & & & & 5 & 1 & & & & & & & & & & & & & \\
\hline & & Helobdella $s p$ & & & & & & & & & & & 1 & & & & & & & & & & & & & \\
\hline Tricladida & Planariidae & Planariidae & & & 1 & & & & & & & & 7 & & & & & & & & & & & & & \\
\hline \multirow[t]{6}{*}{ Trichoptera } & Hydrobiosidae & Atopsyche $s p$ & & 2 & & 1 & & & & & & & & & & & & & & & & & & 1 & & 1 \\
\hline & Hydropsychidae & Leptonema $s p$ & & 7 & 3 & 10 & & & & & 12 & & 7 & 1 & & & & & 5 & & & & & & & \\
\hline & Hydroptilidae & Hydroptila sp & & & & 1 & & & & & & & & & & 1 & & & & & & & & 2 & & \\
\hline & & Ochtrotrichia $s p$ & & & & & & & & & & 1 & & & & 2 & & & & & & & & 2 & & \\
\hline & Philopotamidae & Chimarra sp & & & & & & & & & & & & & & & & & 1 & & & & & & & \\
\hline & Glossossomatidae & Mortoniella $s p$ & 14 & 42 & 27 & 59 & & & 1 & & 8 & 16 & 6 & 46 & 1 & & 1 & 2 & & 9 & 3 & 5 & & 17 & 58 & 12 \\
\hline Tricladida & Naididae & Dugesia sp & & 1 & & 2 & & & & & 1 & 5 & & 3 & & 1 & 6 & 1 & & 6 & 3 & 4 & 3 & 10 & & 3 \\
\hline & SUBTOTAL & & 15 & 90 & 109 & 138 & 6 & 2 & 6 & 3 & 40 & 68 & 58 & 117 & 7 & 8 & 30 & 30 & 26 & 35 & 29 & 17 & 10 & 66 & 97 & 37 \\
\hline & OTAL INDIVIDUOS & & & 352 & & & & 17 & & & & 283 & & & & 7 & 75 & & & 107 & 77 & & & 210 & & \\
\hline
\end{tabular}


Tabla 4. Valores de los índices comunitarios, número de taxas e individuos y BMWP.

\begin{tabular}{|c|c|c|c|c|c|c|c|}
\hline \multicolumn{2}{|c|}{$\begin{array}{l}\text { ESTACIÓN Y FECHA } \\
\text { DE MUESTREO }\end{array}$} & \multirow{2}{*}{$\begin{array}{c}\begin{array}{c}\text { Número } \\
\text { de taxa }\end{array} \\
2\end{array}$} & \multirow{2}{*}{$\begin{array}{c}\begin{array}{c}\text { Número } \\
\text { de indivi- } \\
\text { duos }\end{array} \\
15 \\
\end{array}$} & \multirow{2}{*}{$\begin{array}{c}\text { Diversidad } \\
\text { Shannon- } \\
\text { Weaver }\end{array}$} & \multirow{2}{*}{$\begin{array}{c}\begin{array}{c}\text { Dominancia } \\
\text { Simpson }\end{array} \\
0,12 \\
\end{array}$} & \multirow{2}{*}{$\begin{array}{c}\begin{array}{c}\text { Equidad } \\
\text { de } \\
\text { Pielou }\end{array} \\
0,35 \\
\end{array}$} & \multirow{2}{*}{$\begin{array}{c}\text { BMWP/col } \\
8\end{array}$} \\
\hline \multirow{4}{*}{$\begin{array}{c}\text { E1 San } \\
\text { lorenzo } \\
\text { antes } \\
\text { derivación }\end{array}$} & $\begin{array}{c}\text { Noviembre } \\
\text { de } 2018\end{array}$ & & & & & & \\
\hline & $\begin{array}{c}\text { febrero de } \\
2019\end{array}$ & 12 & 90 & 1,76 & 0,73 & 0,71 & 66 \\
\hline & $\begin{array}{c}\text { Marzo de } \\
2019\end{array}$ & 10 & 109 & 1,65 & 0,75 & 0,71 & 50 \\
\hline & $\begin{array}{c}\text { Mayo de } \\
2019\end{array}$ & 15 & 138 & 1,81 & 0,75 & 0,67 & 73 \\
\hline \multirow{4}{*}{$\begin{array}{c}\text { E2 San } \\
\text { lorenzo } \\
\text { corredor } \\
\text { hiporréico }\end{array}$} & $\begin{array}{c}\text { Noviembre } \\
\text { de } 2018\end{array}$ & 4 & 6 & 1,33 & 0,72 & 0,96 & 21 \\
\hline & $\begin{array}{c}\text { febrero de } \\
2019\end{array}$ & 2 & 2 & 0,69 & 0,50 & 1,00 & 8 \\
\hline & $\begin{array}{c}\text { Marzo de } \\
2019\end{array}$ & 2 & 6 & 0,45 & 0,28 & 0,65 & 15 \\
\hline & $\begin{array}{l}\text { Mayo de } \\
2019\end{array}$ & 3 & 3 & 1,10 & 0,67 & 1,00 & 19 \\
\hline \multirow{4}{*}{$\begin{array}{l}\text { E3 San } \\
\text { lorenzo } \\
\text { salida } \\
\text { truchera }\end{array}$} & $\begin{array}{c}\text { Noviembre } \\
\text { de } 2018\end{array}$ & 10 & 40 & 1,80 & 0,79 & 0,78 & 43 \\
\hline & $\begin{array}{c}\text { febrero de } \\
2019 \\
\end{array}$ & 11 & 68 & 1,99 & 0,82 & 0,83 & 45 \\
\hline & $\begin{array}{c}\text { Marzo de } \\
2019\end{array}$ & 12 & 58 & 1,97 & 0,80 & 0,79 & 54 \\
\hline & $\begin{array}{l}\text { Mayo de } \\
2019\end{array}$ & 12 & 117 & 1,73 & 0,75 & 0,69 & 55 \\
\hline \multirow{4}{*}{$\begin{array}{l}\text { E4 San } \\
\text { lorenzo } \\
\text { claro }\end{array}$} & $\begin{array}{c}\text { Noviembre } \\
\text { de } 2018\end{array}$ & 3 & 7 & 0,80 & 0,45 & 0,72 & 13 \\
\hline & $\begin{array}{c}\text { febrero de } \\
2019\end{array}$ & 7 & 8 & 1,91 & 0,84 & 0,98 & 35 \\
\hline & $\begin{array}{c}\text { Marzo de } \\
2019\end{array}$ & 8 & 30 & 1,74 & 0,79 & 0,84 & 36 \\
\hline & $\begin{array}{c}\text { Mayo de } \\
2019\end{array}$ & 9 & 30 & 1,85 & 0,79 & 0,84 & 38 \\
\hline \multirow{4}{*}{$\begin{array}{l}\text { E5 San } \\
\text { Lorenzo } \\
\text { afluente }\end{array}$} & $\begin{array}{c}\text { Noviembre } \\
\text { de } 2018\end{array}$ & 7 & 26 & 1,33 & 0,62 & 0,69 & 50 \\
\hline & $\begin{array}{c}\text { febrero de } \\
2019\end{array}$ & 5 & 35 & 1,54 & 0,78 & 0,96 & 34 \\
\hline & $\begin{array}{c}\text { Marzo de } \\
2019\end{array}$ & 7 & 29 & 1,69 & 0,78 & 0,87 & 25 \\
\hline & $\begin{array}{c}\text { Mayo de } \\
2019\end{array}$ & 7 & 17 & 1,76 & 0,80 & 0,90 & 33 \\
\hline \multirow{4}{*}{$\begin{array}{l}\text { E6 San } \\
\text { Lorenzo } \\
\text { Unión }\end{array}$} & $\begin{array}{c}\text { Noviembre } \\
\text { de } 2018\end{array}$ & 3 & 10 & 1,03 & 0,62 & 0,94 & 16 \\
\hline & $\begin{array}{c}\text { febrero de } \\
2019\end{array}$ & 10 & 66 & 1,94 & 0,83 & 0,84 & 37 \\
\hline & $\begin{array}{c}\text { Marzo de } \\
2019\end{array}$ & 7 & 97 & 0,94 & 0,53 & 0,48 & 37 \\
\hline & $\begin{array}{c}\text { Mayo de } \\
2019\end{array}$ & 7 & 37 & 1,49 & 0,71 & 0,77 & 40 \\
\hline
\end{tabular}

Con respecto al índice de Diversidad Shannon Weaver encontramos valores bajos, con resultados cercanos a la unidad durante todos los muestreos y en todas las estaciones. La estación San Lorenzo antes de derivación, 
presentó el valor más bajo durante el mes de noviembre; mientras que la estación San Lorenzo salida de truchera, presentó el valor más alto durante el mes de febrero para este índice. Para el índice de Equidad de Pielou su distribución indicó una tendencia uniforme por medio de los valores cercanos a uno (1) a excepción de la estación San Lorenzo antes de derivación en donde el valor es de 0,35 para el mes de noviembre de 2018. En general, el índice nos mostró que la mayor parte de las especies son igualmente abundantes como se muestra en la Tabla 4.

En relación con los índices comunitarios se presentaron valores bajos respecto al número de taxa, el cual oscilo entre 2 y 15. La estación E2 (estación corredor hiporréico) evidenció los menores valores para el número de taxa (2-3), el número de individuos (2-6), el índice de diversidad de Shannon y el BMWP/Col.

La estación E1 es la estación en la parte más alta que se evaluó del río, además que se ubicó antes de la zona de la derivación del sistema hídrico; en esta zona el Río San Lorenzo presentó los mayores valores numéricos de taxa e individuos, índice de diversidad, equidad y del BMWP/Col.

El índice BMWP/Col mostró los puntajes más altos en la estación E1 que evidencia una calidad de aguas moderadamente contaminadas para los meses de noviembre, febrero y marzo, y unas aguas ligeramente contaminadas para el mes de mayo; comparada con las demás estaciones, nos muestra una calidad de agua menos contaminada, contrario a la estación E2 San Lorenzo corredor hiporréico en donde los puntajes registraron valores generales de aguas fuertemente contaminadas. Aunque hay que aclarar que este bajo valor más que indicar contaminación orgánica, es reflejo de la ausencia de flujo, por la desconexión al río.

Las demás estaciones, presentaron puntajes que indican calidad de aguas moderadamente contaminadas y muy contaminadas, las cuales generan dificultades para su utilización como posible fuente abastecedora para el acueducto del municipio de El Carmen de Viboral.

En la tabla 5 se evidencian las variables fisicoquímicas y el porcentaje de varianza explicado para cada uno de los componentes principales, en negrilla se resaltan las relaciones estadisticamente significativas. En el eje 1 se observa que la concentración de oxígeno disuelto y su saturación presentó los valores positivos más altos, mientras que la conductividad eléctrica y los sólidos totales los mayores valores negativos. En el eje 2 los valores de los vectores son bajos y ninguno presento significancia estadística. No obstante, el modelo planteado alcanza una explicación del $40 \%$ de la varianza de la comunidad.

Tabla 5. Variables seleccionadas para el análisis multivariado.

\begin{tabular}{|l|l|l|}
\hline Variable & Eje 1 & Eje 2 \\
\hline $\mathrm{pH}($ unidades de $\mathrm{pH})$ & 0,60 & 0,01 \\
\hline Oxígeno disuelto $(\mathrm{ppm})$ & $\mathbf{0 , 7 0}$ & $-0,06$ \\
\hline Saturación de oxígeno $(\%)$ & $\mathbf{0 , 7 2}$ & $-0,12$ \\
\hline Temperatura del agua $\left({ }^{\circ} \mathrm{C}\right)$ & 0,05 & 0,20 \\
\hline Conductividad eléctrica $(\mu \mathrm{S} / \mathrm{cm})$ & $\mathbf{- 0 , 7 8}$ & $-0,19$ \\
\hline Sólidos Totales Disueltos $(\mathrm{mg} / \mathrm{l})$ & $\mathbf{- 0 , 7 9}$ & $-0,18$ \\
\hline NO3 $(\mathrm{mg} / \mathrm{l})$ & 0,57 & $-0,14$ \\
\hline NO2 $(\mathrm{mg} / \mathrm{l})$ & 0,02 & $-0,17$ \\
\hline $\mathrm{PO} 4(\mathrm{mg} / \mathrm{l})$ & $-0,18$ & $-0,3$ \\
\hline Fe $(\mathrm{mg} / \mathrm{l})$ & $-0,39$ & $-0,13$ \\
\hline
\end{tabular}


El RDA generó un porcentaje de varianza total explicado del $40 \%(p=0.02)$, por lo tanto, se sugiere que la correlación entre los macroinvertebrados y las variables ambientales fue significativa. Para este análisis se usaron 10 variables (Tabla 5). El RDA evidencia que entre las variables fisicoquímicas de mayor peso para la comunidad está la conductividad eléctrica, la concentración de oxígeno disuelto, los sólidos totales disueltos, los nitratos y el fósforo soluble reactivo.

Las variables cuyos vectores presentaron mayor tamaño evidencian posiciones opuestas, mostrando una relación inversa entre la concentración de sólidos totales disueltos, el hierro, la conductividad eléctrica y los ortofosfatos, respecto a la concentración de oxígeno disuelta-porcentaje de saturación, nitratos y pH. La temperatura del agua presentó un patrón diferente al anterior, en el que se relacionan con el primer muestreo.

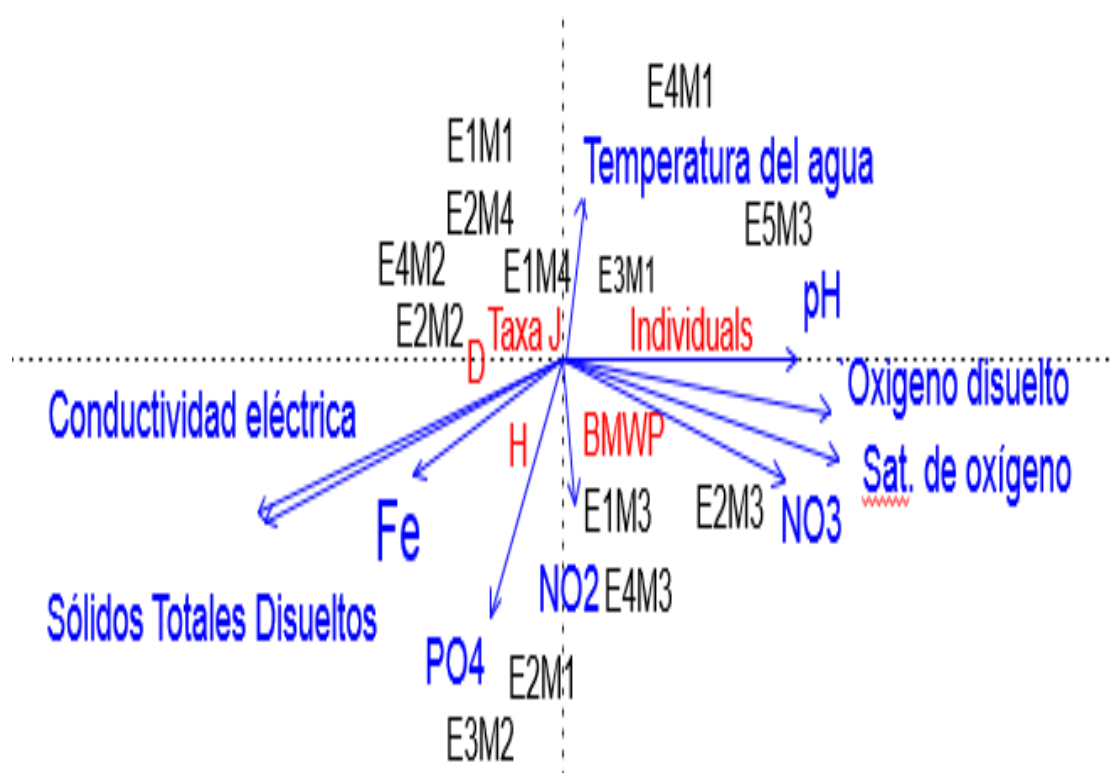

Figura 3. RDA triplot entre la comunidad de macroinvertebrados, variables ambientales y estaciones y momentos de muestreo. Los puntos representan las seis estaciones y cuatro muestreos, las líneas azules simbolizan las variables ambientales y los íconos rojos los índices comunitarios.

El índice BMWP/Col se asoció con la concentración de nitratos y de oxígeno disuelto. En forma inversa con la conductividad eléctrica, los sólidos totales y la concentración de fósforo; los índices comunitarios se ubicaron en el centro del grafico por lo que no evidenciaron relaciones marcada con las variables ambientales medidas.

Al graficas los valores promedios del BMWP/Col para las estaciones de muestreo (Figura 4) evidencia que las condiciones del Río San Lorenzo pese a estar poco poblada su rivera, evidencia contaminación orgánica, evidenciándose que en la zona de la estación1 sin intervención las aguas ya registran un grado leve de contaminación, la cual se acentúa en la E3 después de recibir la descarga de las aguas de la truchera, las cuales no tienen tratamiento previo. El afluente que recibe el río en la E5 presenta una turbiedad marcada por el transporte de sedimentos, lo que disminuye la biota de macroinvertebrados, pero puede generar dilución de la carga de nutrientes, tal así que en la E6 el sistema logra estabilizar la carga orgánica y vuelve a registrar las condiciones de calidad biológicas que tenía antes de recibir las aguas de las trucheras. 


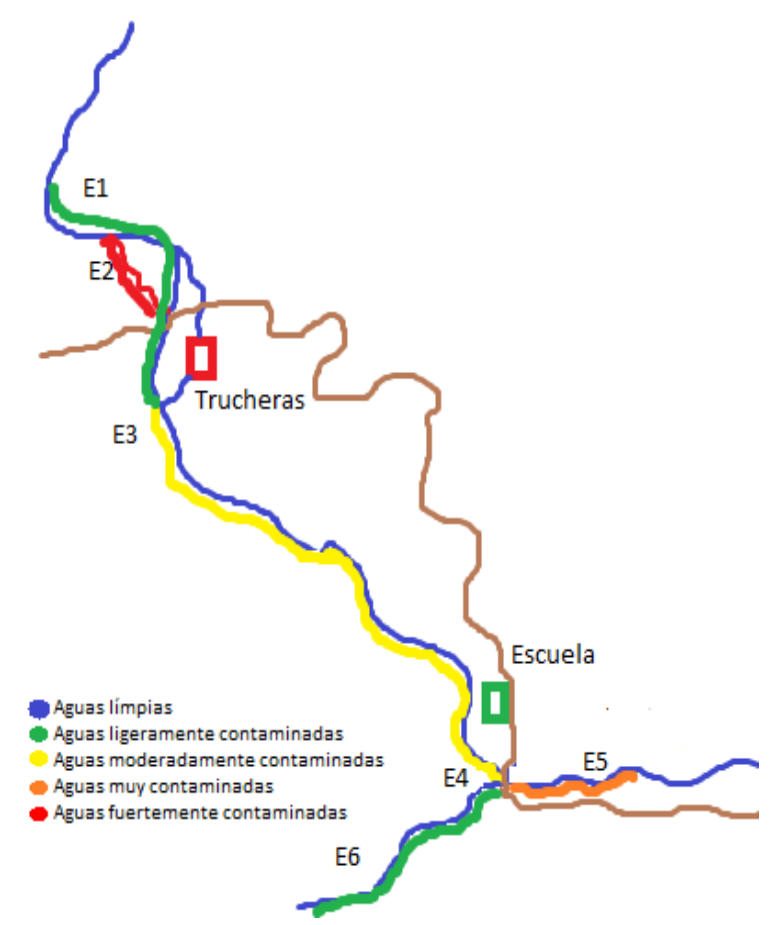

Figura 4. Variación de la calidad biológica del agua por medio de píndice BMWP/Col.

La estación E2 se excluye del análisis anterior, ya que más que contaminación, evidencia una desconexión del sistema fluvial, la cual pese a los 20 años que han pasado, interrumpe la continuidad del río.

\section{DISCUSIÓN}

La desviación del Río San Lorenzo para apresar su caudal con el objetivo de derivar sus aguas para el establecimiento de estanques para truchas, supuso la reducción del caudal circulante lo que pudo llegar a retraer el caudal del sistema pluvial, dejando el curso seco en un tramo de 100m. Dichas actividades sumadas a otras acciones de origen antrópico, afectan la dinámica de los ecosistemas acuáticos [15]; [16]; [17]; [18]; [19];[20].

La temperatura en el Río San Lorenzo presentó un valor promedio de $16,3{ }^{\circ} \mathrm{C}$, valor que favorece mantener niveles óptimos de oxígeno disuelto. Esta variable es de suma importancia debido a que, en condiciones de alta temperatura, disminuye el oxígeno disuelto; aumentando la actividad bacteriana y la sensibilidad de la biota acuática a ciertos componentes tóxicos [5].

La concentración de oxígeno disuelto tuvo valores promedio subsaturados, con un valor medio cercano a $7 \mathrm{mg} / \mathrm{L}$ y con rango ligeramente inferior, cuyo valor menor a $4 \mathrm{mg} / \mathrm{L}$ se encontró en la $\mathrm{E} 2$, lo que indica un nivel adecuado para la vida acuática [8], para el caso de peces se establece que debe ser mayor a $4 \mathrm{mg} / \mathrm{L}$ [21]. Estos autores afirman que este valor está relacionado con los valores de nitrato y fosfato que para el caso del Río San Lorenzo se registra un nivel de nitrato de $0,90 \mathrm{mg} / \mathrm{L}$ valores cercanos al límite inferior de las aguas tropicales colombianas, lo que indica unos niveles bajos frente al uso de agroquímicos y vertimientos domésticos que generalmente son ricos en nitrógeno. La concentración de fosfato registró un valor de 1,33 $\mathrm{mg} / \mathrm{L}$ que indicarían un ambiente mesotrófico; valores superiores están asociados con factores como materia orgánica proveniente de vegetación rivereña, descargas sólidas y líquidas de actividades domésticas y agrícolas. Las concentraciones de fosfato en el agua actúan como nutriente que favorece el alto crecimiento de algas y causa eutrofización. 
El pH en el Río San Lorenzo, presentó un valor promedio de 6,96, que se encuentra dentro de los límites permisibles establecidos en la calidad del agua potable que debe estar entre 6,5 y 9,0 [5]. Según el autor, este criterio es fundamental debido a que puede originar alteraciones en la composición faunística y florística de los cuerpos de agua; así como un aumento de la influencia de ciertos compuestos tóxicos. Desde el punto de vista de los valores de las aguas naturales, los valores registrados en estas investigaciones son normales [21].

La conductividad eléctrica presentó un valor promedio de $21,7 \mu \mathrm{S} / \mathrm{cm}$, muy por debajo del rango establecido para ríos de montaña que oscilan entre $30-60 \mu \mathrm{S} / \mathrm{cm}$ [5]. Resultados inferiores a estos valores indican baja productividad [20].

Los sólidos totales disueltos (STD) presentaron un valor promedio de 22,1 mg/L estando por debajo de los límites establecidos para hábitats acuáticos cuyo valor es de $100 \mathrm{mg} / \mathrm{L}$. Bajas concentraciones favorecen la disponibilidad de oxígeno.[5]; [21].

En el Río San Lorenzo los macroinvertebrados más abundantes son del orden Trichoptera, familia Glossossomatidae. Este orden se caracteriza por tener taxas que normalmente son intolerantes a la contaminación por lo que su número poblacional disminuye con el aumento de esta [22]. Esta familia no se reportó en la E2, probablemente debido a que al ser constructores de casas requieren granos de arena para tal fin y la ausencia de flujo limita al máximo el arrastre de este tipo de material.

Las familias Baetidae y Leptophlebiidae pertenecientes al orden Ephemeroptera, Hydropsychidae del orden Trichoptera y Chironomidae del orden Díptera son las familias que registran una mayor distribución espacio temporal. La familia Leptophlebiidae se destaca por el género Thraulodes. La distribución de esta familia en aguas naturales se considera limitada por su alta sensibilidad a alteraciones ambientales [23]. ,[24]. Esta sensibilidad a la contaminación se evidenció con su presencia en cinco de las seis estaciones de muestreo.

La familia Hydropsychidae se colectaron individuos del género Leptonema sp en las estaciones San Lorenzo antes de derivación y salida de trucheras. Las taxas pertenecientes a esta familia se encuentran en todo tipo de corrientes, utilizan piedras, troncos y hojarasca para construir refugios y se relacionan con altos niveles de oxígeno [14], aunque pueden soportar cierto grado de contaminación orgánica.

La familia Chironomidae se caracterizó por la abundancia y diversidad de dípteros en las seis estaciones de muestreo, aunque en la estación E2 corredor hiporréico es la de menor número de individuos recolectados. [25] mencionan que es una familia de los grupos de dípteros con mayor diversidad y abundancia, condiciones importantes que permiten identificar el amplio papel que cumplen en el funcionamiento de un reoambiente.

Los resultados obtenidos tras la aplicación del índice BMWP/Col e índices comunitarios indicaron el deterioro de la calidad del agua, especialmente en la Estación E2, ya que la ausencia de flujo y la desconexión con el cauce principal limita los procesos de colonización y deriva de los macroinvertebrados acuáticos, entre los cuales se observa una sustitución de los taxones característicos de aguas de cabecera (Tanytarsini, Hetaerina americana, Acari, varios géneros de tricópteros como Atopsyche, Leptonema e Hydroptila) por un grupo de taxones más tolerantes como moluscos (Ferrisia sp y Physa sp) y una reducción del más del $85 \%$ de la riqueza de taxa.

La composición de macroinvertebrados en los diferentes períodos hidrológicos no presentó diferencias significativas, esto puede estar relacionado al hecho de que en la región tropical las estaciones climáticas no son muy marcadas. Resultados similares fueron encontrados por [26] quienes plantearon que estos resultados pueden deberse a la falta de réplicas que permitan hacer comparaciones entre periodos climáticos.

Pese a que el cambio del cauce se realizó hace más de 20 años, el agua que conforma el corredor hiporréico evidencia diferencias en riqueza, abundancia de macroinvertebrados acuáticos y de calidad biológica del agua respecto a las aguas del Río antes del cambio de cauce. La zona hiporréica (HZ, Hyporheic Zone) es una zona 
donde se dan importantes procesos de intercambio de solutos y transformación de nutrientes. Su existencia ha sido ampliamente documentada y estudiada tanto en ríos de montaña, como en ríos de planicie. Sin embargo, en Colombia no se ha realizado casi estudios de caracterización de la zona hiporréica en corrientes naturales [27].

Otro aspecto a mencionar tiene que ver con la reducción del bosque rivereño y la construcción de un "tapón" de cemento que impide que el agua fluya por su antiguo cauce. La vegetación de ribera desempeña un papel importante en la preservación del recurso hídrico y en la estabilización de los cauces; actúa como corredor de dispersión de la biota y como albergue para la fauna en época seca, además las riberas constituyen un sistema de redes interconectadas con el resto de ecosistemas adyacentes (corredor bioecológico), por lo cual su conservación y restauración no se pueden considerar de manera aislada, sino a escala de cuenca hidrográfica [28]; [29].

La alteración del cauce de un río reduce considerablemente el aporte de material alóctono y autóctono lo que afecta la disponibilidad de alimentos, material de refugio, variación de las condiciones fisicoquímicas del agua respecto al cauce principal, incluso se evidenció una colonización excesiva de las rocas por parte del perifiton, especialmente algas verdes filamentosas.

Se observó que en la estación E2 la intervención realizada hace 20 años sigue impactando el cuerpo de agua remanente, el cual según [30] no ha logrado recuperarse, ya que no contiene suficientes recursos bióticos y abióticos como para continuar su desarrollo sin ayuda o subsidio adicional. Este ecosistema se mantiene tanto estructural como funcionalmente simplificado y no demuestra capacidad de recuperación dentro de los límites normales de estrés y alteración ambiental. Presenta poca interacción con ecosistemas contiguos en términos de flujos bióticos y abióticos e interacciones culturales.

\section{CONCLUSIONES}

Pasados 20 años después de ser intervenido un ecosistema se esperaría que tienda a restaurarse por sí solo (resiliencia, adaptación al disturbio, disipación de la entropía); en el caso del tramo del Río San Lorenzo en la estación E2 corredor hiporréico, el Río no alcanzo a restaurarse debido al poco flujo hidrológico por este tramo. La poca biodiversidad encontrada en el agua, es evidencia de las malas condiciones de la misma.

La poca presencia de macroinvertebrados en el tramo intervenido por la derivación del Río San Lorenzo, es el resultado de las condiciones actuales de parte de un ecosistema acuático que no ha logrado restablecerse y que en algún momento fue parte de un sistema dinámico estable. A la luz del BMWP este tramo presenta condiciones críticas.

Las condiciones fisicoquímicas del agua reflejan una baja mineralización, lo cual puede ser indicador de unos suelos meteorizados con bajo impacto antrópico; respecto a la concentración de nutrientes, los fosfatos presentan valores por encima del promedio de la zona, lo que refleja el impacto de la industria de la trucha en el río.

El municipio de El Carmen de Viboral debe velar por el cuidado de este río, por lo que debe requerir a los propietarios de la truchera para que realicen un plan de manejo de la misma y de forma urgente, al menos, generar un sistema de tratamiento básico que limite la salida de nutrientes de la truchera al río San Lorenzo.

En segunda instancia, se debe hacer un seguimiento a la identificación de otras fuentes de contaminación, para implementar pozos sépticos y seguir reduciendo los aportes externos al río. También se recomienda continuar con el monitoreo de la calidad del agua del rio San Lorenzo para verificar si las medidas tomadas se reflejan en un mejoramiento de las condiciones fisicoquímicas y biológicas del agua. 


\section{AGRADECIMIENTOS}

A la Universidad Pontifica Bolivariana a través de la Facultad de Ingeniería y el programa de posgrado, por el apoyo y acompañamiento durante el proceso formativo.

A la Universidad de Antioquia a través del Grupo de Investigación GeoLimna y al Municipio de El Carmen de Viboral por la financiación del proyecto BIOPROSPECCIÓN DE LOS RÍOS SAN LORENZO, GUARINÓ Y COCORNÁ, MUNICIPIO DE EL CARMEN DE VIBORAL, ANTIOQUIA, que dio origen a este trabajo.

\section{REFERENCIAS BIBLIOGRÁFICAS}

[1] CORTOLIMA. (2004). II Fase Diagnostico - Río Coello. L-2.11 Calidad de Aguas. 111(479), 1009-1010.

[2] Conesa, C., \& Pérez, P. (2014). Alteraciones geomorfológicas recientes en los sistemas fluviales mediterráneos de la península ibérica. Síntomas y problemas de incisión en los cauces. Revista de Geografía Norte Grande, 2014(59), 25-44.

[3] Roldán P., G. (1988). Guía para el estudio de los Macroinvertebrados acuáticos del departamento de Antioquia. 217p. Medellín: Universidad de Antioquia.

[4] Ballarin, D., \& Rodríguez, I. (2013). Hidromorfología fluvial. 126.

[5] Gualdrón, L. E. (2018). Evaluación de la calidad de agua de ríos de Colombia usando parámetros fisicoquímicos y biológicos. Dinámica Ambiental, (1), 83-102. Disponible en: https://doi.org/10.18041/25906704/ambiental.1.2016.4593 [consultado el 30 de agosto de 2019].

[6] Roldán, G. (2003). Bioindicación de la calidad del agua en Colombia: propuesta para el uso del método BMWP/ Col. (p. 142). p. 142. Medellín: Universidad de Antioquia.

[7] González, S., Ramírez, Y., Mesa, A. M., \& Dias, L. (2012). Diversidad De Macroinvertebrados Acuáticos Y Calidad De Agua De Quebradas Abastecedoras Del Municipio De Manizales. Boletín Científico. Centro de Museos. Museo de Historia Natural, 16(2), 135-148.

[8] Bustamante, P., \& Angulo, A. M. (2018). Prevalencia de las familias de Macroinvertebrados como Bioindicadores de la calidad de agua en dos ecosistemas del Ecuador continental. Escuela Superior Politécnica de Chimborazo. Orellana.

[9] Roldán, G. (2016). Los macroinvertebrados como bioindicadores de la calidad del agua: cuatro décadas de desarrollo en Colombia y Latinoamérica. Revista de La Academia Colombiana de Ciencias Exactas, Físicas y Naturales, 40(155), 254.

[10] CORNARE. (2006) Subregión Valles de San Nicolás. Disponible:

http://www.cornare.gov.co/contenidos.php?seccion=2\&id=2. [Consultado: 28 de enero 2006]

[11] McCaffertty, W. P. Aquatic Entomology. Boston: Science Books International. 1981.

[12] Merritt, R. y K. Cummins. An Introduction to the Aquatic Insects of North America. Dubuque: Kendall/Hunt Publishers. 1996.

[13] Álvarez, L. F. (2006). Metodología para la utilización de los macroinvertebrados acuáticos como indicadores de la calidad del agua. Instituto Alexander von Humboldt.

[14] Dominguez, E., \& Fernández, H. R. (2009). Macroinvertebrados bentónicos sudamericanos. Sistemática y biología ISBN (Vol. 1975). Tucumán Argentina: Fundación Miguel Lillo. 
[15] Obando, N. L., \& Bustamante, C. A. (2014). Macroinvertebrados y algas perifíticas de la quebrada Cajones, unidad de manejo de cuenca UMC Río Espejo municipio de Montenegro, Quindío, Colombia. Revista de La Asociación Colombiana de Ciencias Biológicas, 26, 133-144.

[16] Morales, S., Salazar, M. D. R., \& Urrea, J. D. (2016). Composición y estructura de la comunidad de macroinvertebrados y perifiton del río Ejido, Popayán - Cauca. Revista de Ciencias, 20(2), 16. Disponible en: https://doi.org/10.25100/rc.v20i2.4670 [consultado el 28 de agosto de 2019].

[17] Arias, L. M., Duque, A., Meneses, S., \& Ramírez, J. E. (2016). Estrategias de mitigación de impactos ambientales implementadas en el proyecto hidroeléctrico de Sogamoso. The British Journal of Psychiatry, 111(479), 1009-1010. Disponible en: https://doi.org/10.1192/bjp.111.479.1009-a [consultado el 31 de agosto de 2019].

[18] Ruiz, J. A., Vélez, F. de J., Caicedo, O., \& Aguirre, N. J. (2016). Modelación espacial de la calidad del agua en el río Tapartó, municipio de Andes, Antioquia, Colombia. Revista Mutis, 6(1), 16-27.

[19] Díaz, J. A., \& Granada, C. A. (2018). Efecto de las actividades antrópicas sobre las características fisicoquímicas y microbiológicas del río Bogotá a lo largo del municipio de Villapinzón, Colombia. Revista de La Facultad de Medicina, 66(1), 45-52. Disponible en: https://doi.org/10.15446/revfacmed.v66n1.59728 [consultado el 31 de agosto de 2019].

[20] Escobar, A. F., \& Montoya, Y. (2019). Los macroinvertebrados acuáticos y la calidad biológica del agua en una quebrada andina, Antioquia-Colombia. (p. 15). p. 15. Revista Politécnica.

https://doi.org/10.33571/rpolitec.v15n29a6

[21] Roldán, G., \& Ramírez JJ. Fundamentos de Limnología Neotropical. Editorial Universidad de Antioquia, Medellín. 2008.

[22] Prat, N., Ríos, B., Acosta, R., \& Rieradevall, M. (2009). Los macroinvertebrados como indicadores de la calidad de las aguas. Macroinvertebrados bentónicos sudamericanos. sistemática y biología. 631-654. Disponible en http://www.ub.edu/fem/docs/caps/2009 MacrolndLatinAmcompag0908.pdf [consultado el 30 de agosto de 2019].

[23] Murillo, M., Caicedo, O., Hernández, E., Grajales, H., Mesa, J., Cortés, F., Aguirre, N. (2016). Aplicación de tres índices bióticos en el río San Juan, Andes, Colombia. 6(2), 59-73.

[24] Raimundi, E. (2010). Ecología de Leptophlebiidae bank, 1900 (Insecta: Ephemeroptera). Santa Catarina: No oeste de Santa Catarina, Universidades comunitéria da região de chapecó. Programa de Pós-Graduação em Ciências Ambientais.

[25] Porinchu, D., \& MacDonald, G. (2003). The use and application of freshwater midges (Chironomidae: Insecta: Diptera) in geographical research. Progress in Physical Geography, 27(3), 378-422.

[26] Meza, A.M., Rubio-M, J., Días, L.G. Valteros, J.M. (2012). Calidad de agua y composición de macroinvertebrados acuáticos en la subcuenca alta del río Chinchiná. Caldasia. 34 (2): 443-456.

[27] Amaya-Toro, L. 2016. Identificación de la zona hiporreica en un río sinuoso de montaña. Caso de Estudio Quebrada Aguas Claras, La Calera, Cundinamarca. Trabajo de pregrado Universidad Nacional de Colombia Facultad de Ingeniería. Bogotá.

[28] Naiman, R. J., Bison, P. A., Lee, R. G. \& TURNER, M. (1998). Watershed Management, Chapter 26 (pp. 642-661). En R. J. NAIMAN \& R. E. BILBY (Eds.), River Ecology and Mangement: lesson from the Pacific Coastal Ecoregion. New York: Springer-Verlag.

[29] Guevara, G; Reinoso, G; García, J; Franco, M; García, J; Yara, D; Briñez, N; Ocampo, M; Quintana, M; Pava, D; Flórez, N; Ávila, M; Hernández, E; Lozano, L; Guapucal, M; Borrero, D. y Olaya, E. 2008. Aportes para el análisis de ecosistemas fluviales: una visión desde ambientes ribereños. Revista Tumbaga (2008), 3, 109-127.

[30] Poff, L., Allan, J. D., Bain, M. B., Karr, J. R., Prestegaard, K. L., Richter, B., Sparks, R. \& Stromberg, J. (1997). The natural flow regime: a paradigm for river conservation and restoration. BioScience, 47, 769-784. 